\title{
Induced anisotropy by the Mullins effect in filled silicone rubber
}

\author{
G. Machado, G. Chagnon*, D. Favier \\ Université de Grenoble/CNRS, Laboratoire 3S-R, Cedex 9, 38041 Grenoble, France
}

\begin{abstract}
This study is concerned with the experimental characterization of anisotropy induced by the Mullins effect in a particle-reinforced silicone rubber. Experimental data concerning the influence of type and direction of initial loading on the subsequent stress softening are quite scarce. In this scope, a set of experimental tests were carried out on a filled silicone rubber. Uniaxial tensile tests and bulge tests were used to precondition the samples, i.e., to induce some primary stress softening. In both cases, subsequent uniaxial tensile tests were conducted on preconditioned specimens. The first set of experiments consists of a uniaxial tension path followed by uniaxial tension along different directions. It appears that the stress softening varies from a maximum in the same direction load to a minimum in the orthogonal direction, with respect to the first tensile load direction. Next, the bulge test is proposed as an original way to yield very different biaxial tensile strain-histories for first loading path. The fact that the biaxiality ratio varies from the pole (uniaxial tension) until the bulge border (planar tension), permits to analyze second tensile load curves in a material that experienced a more complex first load path. These experimental data allow to discuss the most appropriate criteria to describe the strain-induced anisotropy phenomenon.
\end{abstract}

\section{Introduction}

Rubber-like materials exhibit a significant stress softening after a first loading. This phenomenon called Mullins effect has been observed in different paths of deformation during the last six decades. A detailed review for experimental references in different deformation states like uniaxial tension, uniaxial compression, hydrostatic tension, simple shear and equibiaxial tension is given in Diani et al. (2009). The present work is concerned to study of a filled silicone rubber. Even if the stress softening is more pronounced in filled rubbers (Mullins, 1947), there are evidences to suggest that it can occur in unfilled rubbers (see for references Rigbi, 1980; Johnson and Beatty, 1993).

In a first approach, the Mullins effect was analyzed under the isotropy hypothesis, which has led to numerous models in terms of pseudo elasticity (Ogden and Roxburgh,

\footnotetext{
* Corresponding author.

E-mail address: gregory.chagnon@grenoble-inp.fr (G. Chagnon).
}

1999; Dorfmann and Ogden, 2004), damage modeling (Chagnon et al., 2004) and macromolecular approaches (Govindjee and Simo, 1991; Arruda and Boyce, 1993; Marckmann et al., 2002). More references can be found in Diani et al. (2009). Nevertheless, this softening phenomenon is known to exhibit very strong directional dependency. This phenomenon was first exhibited in Mullins (1947), where the author alluded that the degree of softening for natural rubber was not the same for samples cut along and perpendicular to the direction of the previous preconditioning tensile stretch, and thus some anisotropic stress strain properties were developed. However, the corresponding experimental data have not been published.

More recently the evidence that stress softening is an inherently anisotropic phenomenon can be found for different rubber-like materials under different load cases. However, these investigations are almost restricted to a simple deformation history. Uniaxial tensile tests in two orthogonal directions have been the most used method to point out the strain-induced anisotropy. Results can be seen in 
Laraba-Abbes et al. (2003) for a carbon-black filled natural rubber as material, Diani et al. (2006) for filled black ethylene propylene diene (EPDM), Itskov et al. (2006) for carbonblack filled acrylate rubber (ACM), Hanson et al. (2005) for a silica-filled polydimethylsiloxane (PDMS) and Park and Hamed, (2000) for different compositions of black filled styrene-butadiene rubber (SBR) and sulfur-cured carbon black filled natural rubber (NR). Simple shear experiments were performed by Muhr et al. (1999), they noticed that strain softening of polysiloxane polymer investigated is smaller in directions orthogonal to the strain cycle causing the softening. Similarly under simple shear, Besdo et al. (2003) showed that a prior loading, generated by a cyclic one-side shear, leads to a material anisotropy verified during a symmetric loading, i.e., both-sided shear. Pawelski (2001) carried out two tests, first, a homogeneous plane-strain compression using a cubic sample of an elastomeric material (VLGQ-rubber) rotated and rotated back by $90^{\circ}$ with respect to the first preloaded configuration. Here a special procedure was required to correct the original measurements since compression is accompanied by friction which spoils the experimental data. Second, for a polyurethane material, a modified biaxial tension with uniaxial tensile tests on secondary specimens afterward, was used in a way to find an evidence for more complex memory behavior of the material.

Many physical interpretations for the Mullins effect have been proposed but few give an interpretation of the direction influence of the stress softening. Let us quote Papkov et al. (1975), who investigated the softening of siloxane rubbers. Their investigations suggest that the most probable softening mechanism is not the separation of the polymer molecules from the surface of the filler particles but the rearrangement of the chains on that surface as a result of slippage under stress. In addition, they considered that the transition of a given filled system to the quasi-stable state during the deformation may be accompanied not only by slippage of the chains on the surface of the particle but also by a certain rotation and irreversible displacement of the particle in the polymer matrix. This should lead to a corresponding change in the threedimensional structure of the filled rubber and may be accompanied by the development of anisotropy of the mechanical properties. Hanson et al. (2005) provided another interpretation for the stress softening mechanism, based on the remove of chains entanglements that causes the stress to be lower on the second and subsequent loadings, i.e., their mechanism conserves the number of network chains and only the entanglements density is reduced with respect to the original strain axis of the first load.

Some models aiming to describe the anisotropy of the stress softening are proposed in the literature see for example, (Diani et al., 2004, 2006; Göktepe and Miehe, 2005; Shariff, 2006; Ehret and Itskov, 2009). All these models provide a solid theoretical basis for mathematical description of the stress softening, like an anisotropic phenomenon, in rubber-like materials. However, the predictive capabilities of models describing the anisotropic Mullins effect are mainly restricted to the uniaxial tension case. Horgan et al. (2004) reported that the anisotropic model cannot be validated since suitable experimental data are not available. Pawelski (2001) advised that further experimental works are necessary before one can decide how complex a material constitutive equation for rubberlike material should be. Itskov et al. (2006) pointed out that additional experiments regarding other load cases than uniaxial tension are needed. This allows to study further the influence of loading cycles with complex deformation states on the appearance and evolution of the Mullins effect in the case of changing principal stretch directions.

Within this context, the aim of this work is to quantify clearly the induced anisotropy according to the applied strain history. The objective is to focus on the strain-induced anisotropy study by verifying the influence of loading cycles in distinct directions using two different prestretch methods to drive the primary stress softening history. The paper is organized as follows. In Section 2 the experimental method and results are presented. First, uniaxial tension of a large plate was used to induce the primary deformation. Then, the influence of induced anisotropy was investigated for different directions by cutting different specimens from the original plate and comparing the second uniaxial tensile load curves. Second, the bulge test was used to generate different biaxial tensile deformation histories, and again cut specimens from the original plate were thus submitted to uniaxial tensile tests. In Section 3 a study of generated anisotropy is realized by analyzing the second load curves of the different tests and comparing their strain histories. Finally, Section 4 contains some concluding remarks and outlines some perspectives.

\section{Experimental study and analysis}

The induced anisotropy by stress softening was experimentally studied. For this purpose, samples were made of a filled silicone rubber called Rhodorsil RTV3428. Supplied as two liquid components, the uncured silicone and the curing agent, this liquid mixture is molded by injection to obtain a sheet with constant thickness (Meunier et al., 2008). The final samples are produced by a polyaddition, curing at $70{ }^{\circ} \mathrm{C}$ for $4 \mathrm{~h}$ in order to accelerate the curing process and assure a sufficient cross-linking density. This material exhibits a pronounced stress softening under different load cases, as reported in Machado et al. (2010). In this same work, the manufacture procedure of specimens without any pre-existent anisotropy and testing methods details using three-dimensional image correlation (3DDIC) are also described. With the 3D-DIC technique, it is possible to determine the $3 \mathrm{D}$ contour and the in-plane strain fields of the object surface. This process is carried out by correlation of the images, taken by two cameras in the deformed state with their original reference images. Thus, uniaxial tensile test and bulge test were used to precondition the samples, i.e., to induce some primary stress softening. In both cases, subsequent uniaxial tensile tests are conducted on preconditioned specimens. The first experiment consists in a uniaxial tension path followed by uniaxial tension in different directions. After, the bulge test is used to yield a very different biaxial tensile strainhistories as first load path. 

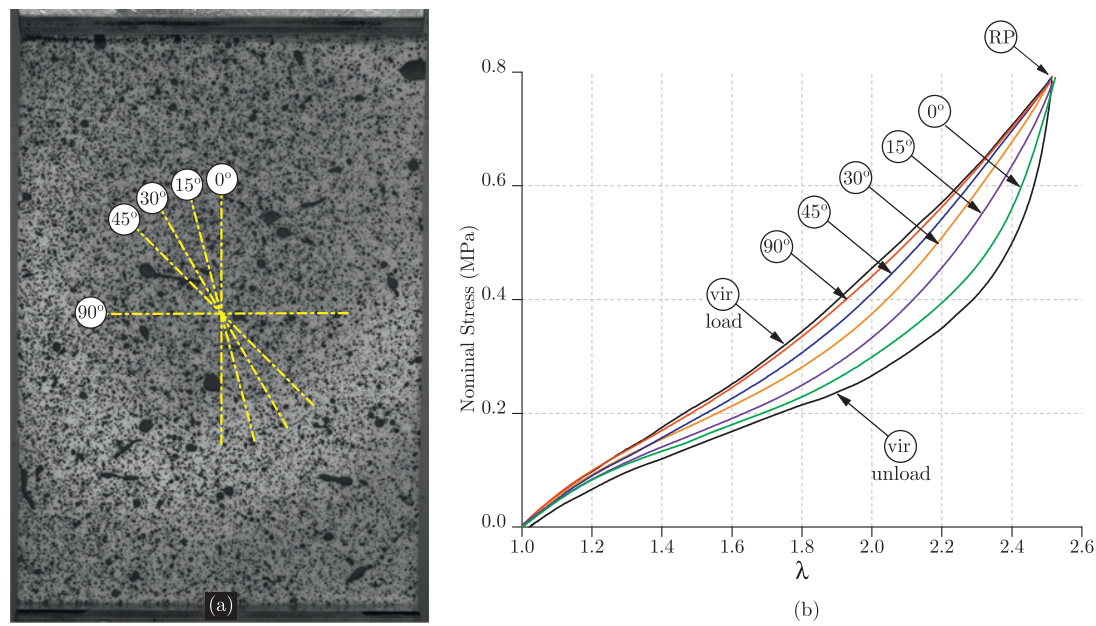

(b)

Fig. 1. (a) Preconditioned large sample with the representation of different orientations of the tensile test cut specimens. (b) Induced anisotropy represented by the superposed uniaxial stress-strain responses for the different angles between the first and second loading directions. Curves are compared with the uniaxial tensile curve (load-unload) of a virgin specimen.

\subsection{Mullins anisotropy induced by uniaxial tensile pre-stretch}

Two identical rectangular specimens (length $l_{0}=100 \mathrm{~mm}$, width $w_{0}=70 \mathrm{~mm}$ and thickness $h_{0}=2 \mathrm{~mm}$ ) were submitted to one cycle of stretch, at $\lambda=2.50$ in uniaxial tension along a principal direction referred to $0^{\circ}$, using an elongation rate of $\dot{\lambda}=5.0 \times 10^{-3} s^{-1}$. Note that RTV3428 behavior can be assumed independent of the rate of deformation (see Machado et al., 2010). Then, a set of smaller specimens (length $l_{0}=60 \mathrm{~mm}$, width $w_{0}=12 \mathrm{~mm}$ and thickness $h_{0}=2 \mathrm{~mm}$ ) was cut from each of these preconditioned large samples along five different directions $0^{\circ}$ (first loading direction), $15^{\circ}$, $30^{\circ}, 45^{\circ}$ and $90^{\circ}$ (orthogonal direction). An illustration is presented in Fig. 1(a). All these cut specimens were submitted to the same pre-conditioning strain state and this was verified thanks to strain field measurements.

Fig. 1(b) presents the virgin material uniaxial tension cycle (vir curve) and second tensile loading curves for the different directions, i.e, the different responses of cut specimens in uniaxial tension. Both samples were stretched until $\lambda=2.5$ at $\dot{\lambda}=1.6 \times 10^{-2} s^{-1}$. All subsamples unload curves are superposed with the virgin reference sample unload curve and, for a clean visualization, they are not presented. A very small residual elongation is observed. Thus the effect of permanent set in the cut samples can be considered as negligible, since they were cut after a recovery time of $1 \mathrm{~h}$.

It clearly appears that all second load curves come back on the first loading curve at the same elongation ( $R P$-point) which is the maximal elongation reached during the uniaxial tensile pre-stretch. However, the main difference is the shapes of the second load curves, the amount of stress softening can be relied to the angle between the first and second loading directions. Note that with the angle increasing, the shapes of the second load curves tend toward the virgin material load curve (vir). The perpendicular direction $\left(90^{\circ}\right)$ is nearly superposed with the virgin curve. This evidence was noticed by Papkov et al. (1975) (for samples with an inferior filler ratio), Park and Hamed (2000); Hanson et al. (2005) and Diani et al. (2006). On the other hand, in Papkov et al. (1975) (for samples with a superior filler ratio), Laraba-Abbes et al. (2003) and Itskov et al. (2006), perpendicular specimens have the same shape of the stress strain virgin curve, but with a pronounced lower modulus. This fact may be attributed to the filler nature, and their mass and volume proportion in the polymeric matrix. As pointed out by Itskov et al. (2010) the character and extent of the anisotropy of the Mullins effect depend on the elastomer type, the kind of filler particles, and the filler percentage.

\subsection{Mullins anisotropy induced by biaxial tensile pre-stretch}

The objective of this section is to propose an experimental test that permits to analyze second uniaxial tensile load curves in a material that experienced a more complex first load path. A biaxial tensile pre-stretch can be used to generate a complex in-plane deformation history, under incompressibility assumption. The complexity of this deformation history is given by the biaxiality ratio $(\mu)$, that can be expressed as a relation between principal in-plane stretches $\left(\lambda_{\max }, \lambda_{\min }\right)$ in the form

$\mu=\frac{\ln \left(\lambda_{\min }\right)}{\ln \left(\lambda_{\max }\right)}$.

Thus, biaxiality ratio is very useful to characterize the first history imprinted in the material at the first loading. Note that in the uniaxial tensile pre-stretch (Section 2.1), the contractive stretches are the minimal principal directions, then the biaxiality ratio is fixed in $\mu=-0.5$.

In this work the bulge test is proposed as an original way to yield very different biaxial tensile strain-histories for first load path. As pointed in Machado et al. (2012) bulge test is able to provide more information than the traditional application to determine the material equibiaxial tension response. It can be used to generate different biaxial tension states along a radial path of specimen from the 


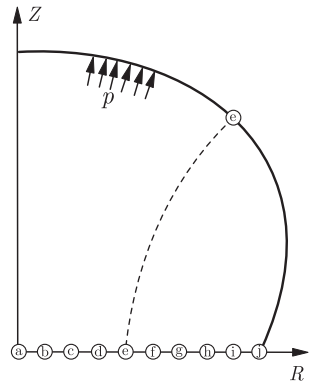

(a)

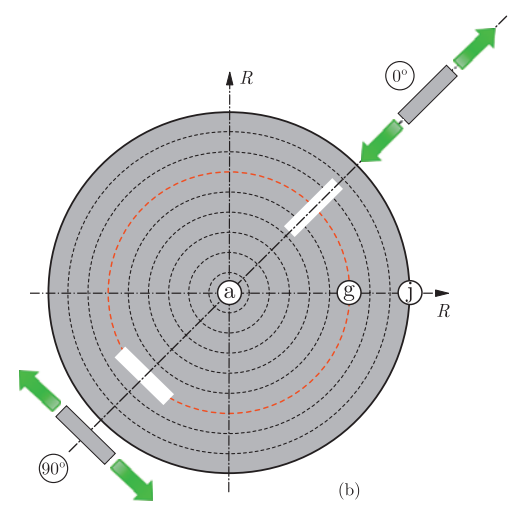

Fig. 2. Schematic representation of biaxial preconditioning method and principal stretches: (a) axisymmetric representation of the bulge test with the interest points; (b) Illustration of the cut subsamples over different directions in the circular membrane.

equibiaxial tension state $(\mu=1)$ at the pole until the planar tension state $(\mu=0)$, i.e., a large biaxiality ratio interval $(\mu \in[0,1])$.

Using the 3D-DIC technique, it is possible to determine the three-dimensional surface displacements and the principal stretches $\left(\lambda_{m}, \lambda_{c}\right)$ in the meridional and circumferential directions, as schematized in Fig. 2. From this point forward, these directions will be denoted by the subscripts $m$ and $c$ respectively. Nevertheless, in the axisymmetric case, deformed membrane principal stresses $\left(\sigma_{m}, \sigma_{c}\right)$ are determined from principal curvatures $\left(\kappa_{m}, \kappa_{c}\right)$ combined with the measurement of the inflating pressure $(p)$. These fields are related as follow

$\sigma_{m}=\frac{p}{2 h \kappa_{c}}$,

$\sigma_{c}=\frac{p}{2 h \kappa_{c}}\left(2-\frac{\kappa_{m}}{\kappa_{c}}\right)$,

where $h$ is the current thickness calculated using the incompressibility assumption. $\kappa_{m}$ and $\kappa_{c}$ are deduced from 3D-DIC measurements. Thus, the stress-strain state is then known at any surface point which enriches greatly experimental data deduced from bulge tests. For a comprehensive explanation see Machado et al. (2012).

The specimen geometry consists in thin circular plate, of initial radius $R=90 \mathrm{~mm}$ with a thickness $h_{0}=2 \mathrm{~mm}$. The circular plate is simply supported and subjected to an uniform inflating pressure acting perpendicular to the current configuration. Using the 3D-DIC strain field measures, a set of interest points are specified along the radius of undeformed membrane permitting to know the stress and strain histories for every point over the deformed membrane surface. This set of points is represented in Fig. 2, where each point can be regarded as a small specimen in view of 3D-DIC as a full field strain measure method. Point $a$ corresponds to the membrane center and the other points ( $b$ to $j$ ) are $10 \mathrm{~mm}$ equidistant through the meridional direction.

Fig. 3 presents the first loading strain and stress history of points $a$ to $h$ in function of the load pressure. Note that each inflation state involves a heterogeneous stress-strain state that evolves from an equibiaxial tensile state at the pole $(R=0 \mathrm{~mm}$ - point $a)$ tending to planar tensile stretching nearest the clamp $(R=90 \mathrm{~mm}$ - point $j)$. Machado et al. (2012) pointed out the experimental difficulty concerning the measurements on the overall membrane surface and the region near the clamp simultaneously. For materials that experience high displacements a self-shadow effect is observed in this last region.

The highest reached values for principal stretches $\left(\lambda_{m}, \lambda_{c}\right)$ and first invariant $\left(I_{1}\right)$ experienced in the prestretch test for each point ( $a$ to $j$ ) are presented in Fig. 4(a). The Fig. 4(b) presents the mean value and the range of the biaxiality ratio $(\mu)$ for all pressure load steps in each point $a$ to $i$. The measured ratio is almost constant at a given point and variations between the different pressure load steps can be disregarded. Due to the experimental difficulties concerning the measurements near the clamp, the curves in Fig. 4 were extrapolated for points $i$ and $j$ using polynomial approximation and represented by dotted lines.

After a recovery time of $1 \mathrm{~h}$, a set of uniaxial tensile test specimens was cut (length $l_{0}=40 \mathrm{~mm}$, width $w_{0}=3 \mathrm{~mm}$ and thickness $h_{0}=2 \mathrm{~mm}$ ). Two series of samples are defined as follows: samples aligned with the meridional direction denoted by $0^{\circ}$ and samples tangent to the circumferential direction (or perpendicular to the meridional direction) denoted by $90^{\circ}$. Both series are illustrated in Fig. 2(b), for instance, at point $g$. The deformation is evaluated locally, using DIC, in a small zone located in the subsamples center.

At first, the samples aligned with the meridional direction $\left(0^{\circ}\right)$ were submitted to a uniaxial tensile test. The loading consists of a simple load unload cycle, where the maximum deformation limit overpasses the maximum reached in the biaxial tensile pre-stretch (see Fig. 4(a)). Fig. 5 presents the second load curves for the different specimens and a virgin uniaxial tensile load curve (vir) as reference. The load curves of specimens $b, c$ and $d$ are quasi superposed, thus they are not represented in the figure keeping clear the visualization of results. Fig. 5 highlights that closer the subsample is from the bulge pole, i.e. when $\mu$ tends to one, higher is the stress softening. However, for points nearest to the border, where $\mu$ tends to zero, the stress softening can be practically disregarded since first 

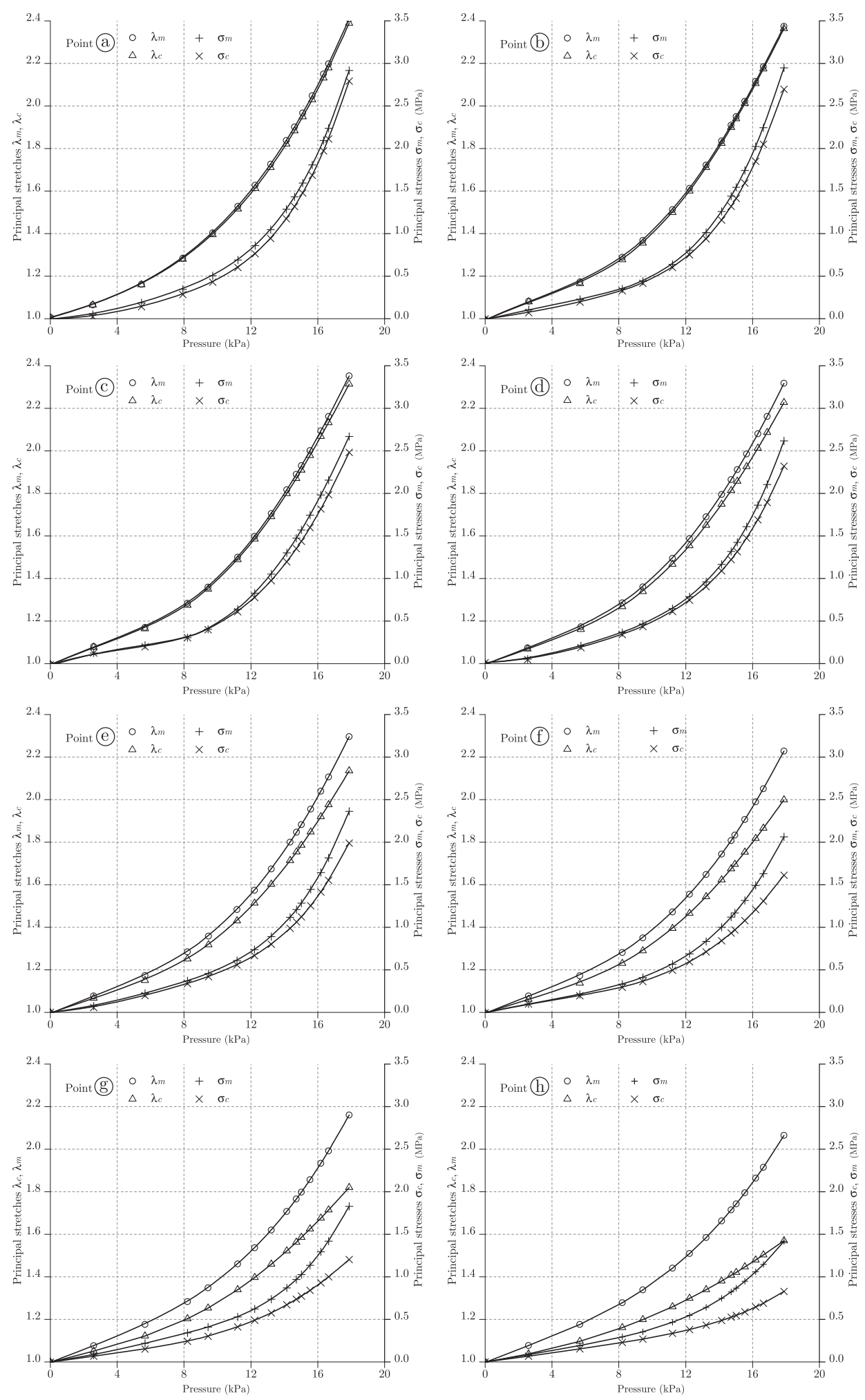

Fig. 3. Bulge test deformation history for points $a$ to $h$. Principal stretches $\left(\lambda_{m}, \lambda_{c}\right)$ and Cauchy principal stresses $\left(\sigma_{m}, \sigma_{c}\right)$ are presented respectively on left and right axis according to the inflating pressure $(p)$. 


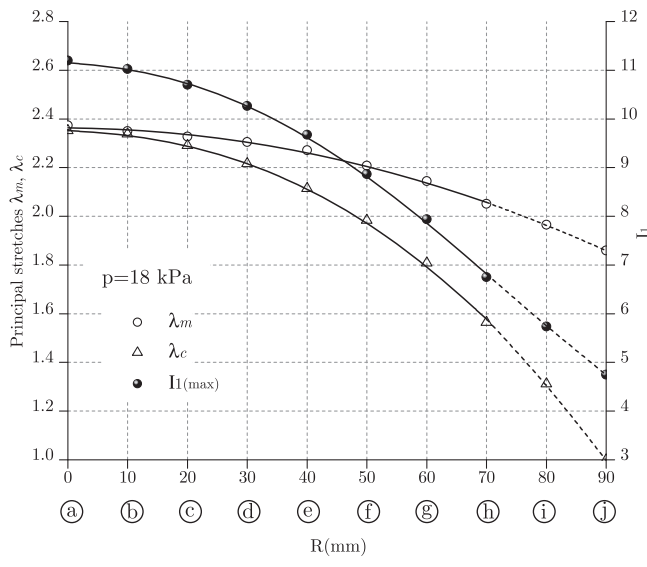

(a)

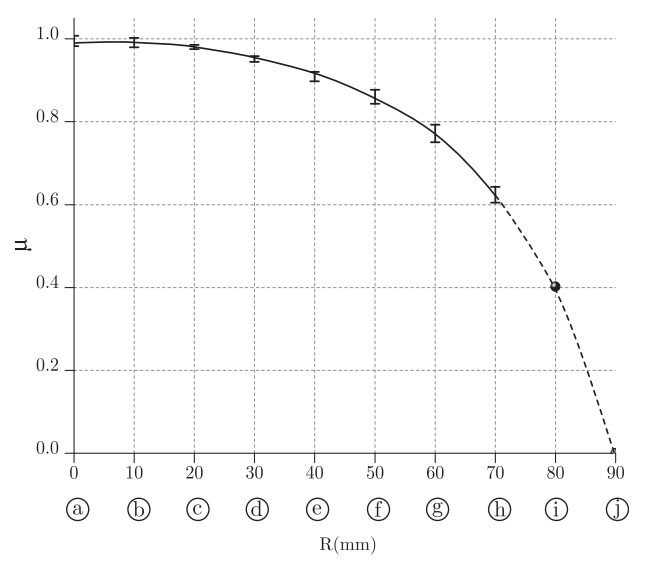

(b)

Fig. 4. (a) Highest deformation level experienced on the first biaxial tensile load ( $p=18 \mathrm{kPa}$ ) for each meridional point ( $a$ to $j$ ) in terms of principal stretches $\left(\lambda_{m}, \lambda_{c}\right)$ and first invariant $\left(I_{1}\right)$; (b) Mean value and range of biaxiality ratio $(\mu)$ during all pressure load steps. Points $i$ and $j$ were extrapolated using a polynomial approximation (dotted lines).

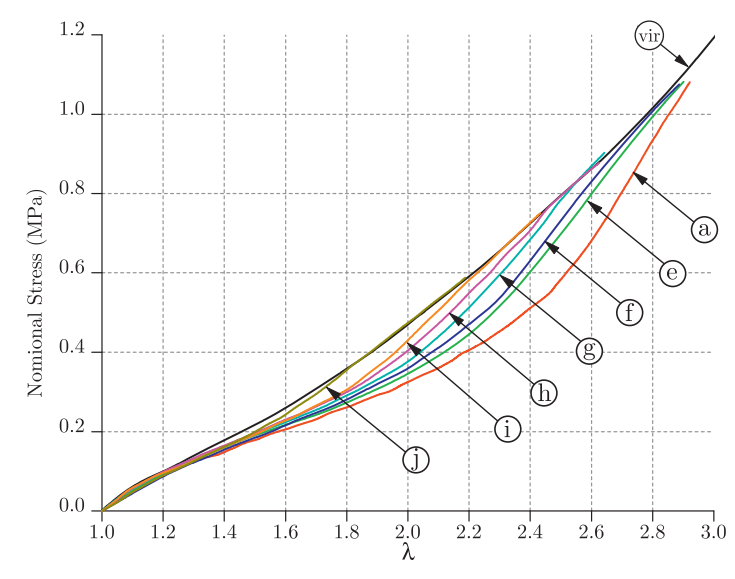

Fig. 5. Influence of the first biaxial tensile load on second uniaxial tensile test response. Uniaxial tension performed in the meridional direction $\left(0^{\circ}\right)$ for the subsamples $a, e, f, g, h, i$, and $j$.

and second loads are quasi-superposed (see point $j$ ). Finally, when $\mu$ evolves from zero to one, the difference between the reference curve (vir) and second load is increasing and the return point $(R P)$ on the reference curve appears for a larger elongation. These results are in consistency with the maximal first deformation $\left(\lambda_{m}\right)$ experienced by the subsamples as presented in Fig. 4(a).

Second, the uniaxial tension samples cut at points $g, h$ and $i$ perpendicularly to the meridional direction (noted as $90^{\circ}$ subsamples) are tested. Results from the $90^{\circ}$ subsamples are therefore compared with the virgin load curve (vir) and also the $0^{\circ}$ subsamples, as presented in Fig. 6. It clearly appears that the second load curves, independently of the second load direction $\left(0^{\circ}\right.$ or $\left.90^{\circ}\right)$, come back on the virgin loading curve (vir) at that same maximal tensile stretch, i.e., the same $R P$. This confirms the results of the previous Section 2.1. However, for a given point the stress softening amounts are different between both directions, except at point $a$ where all directions are equivalent.

\section{Discussion}

\subsection{Anisotropy induced by uniaxial tensile pre-stretch}

First, the uniaxial tension deformation history tests are analyzed. Fig. 7(a) shows the stress ratio $(\delta)$ between the virgin uniaxial tensile load curve $\left(P_{0}\right)$ and the set of subsamples orientated at $0^{\circ}, 15^{\circ}, 30^{\circ}, 45^{\circ}$ and $90^{\circ}$ directions $\left(P_{2^{(\alpha)}}^{(\alpha)}\right)$

$\delta=\frac{P_{2^{\text {nd }}}^{(\alpha)}}{P_{0}}$,

where $P$ denotes the nominal tensile stress. The curves, concerning Eq. (4), highlight that, given a deformation level, there is no stress proportionality between the different directions.

Recalling that the first Piola-Kirchhoff stress tensor $\mathbf{P}$ is work-conjugate to the rate of deformation gradient $\dot{\mathbf{F}}$, a stored strain energy density can be established as the work done by the stresses from the initial to the current position as

$\mathcal{W}=\int_{0}^{t} \mathbf{P}: \dot{\mathbf{F}} d t$

Thus, it becomes possible to define a energy loss ratio $\eta$ that quantifies the evolution of the energy loss by Mullins effect according to the loading angle $\alpha$, given by

$\eta=\frac{\mathcal{W}_{0}-\mathcal{W}_{2^{\text {nd }}}^{(\alpha)}}{\mathcal{W}_{0}-\mathcal{W}_{2^{\text {nd }}}^{\left(0^{\circ}\right.}}$,

where $\mathcal{W}_{0}$ and $\mathcal{W}_{2^{\text {nd }}}^{(\alpha)}$ are respectively the strain energy of virgin material and the strain energy of the second loading in the direction $\alpha$. Both energy quantities are evaluated using Eq. (5) were upper limit is defined at the $R P$ point. Fig. 7(b) presents the evolution of the energy loss by Mullins effect according to the loading angle in the uniaxial tensile pre-stretch condition for the maximal 


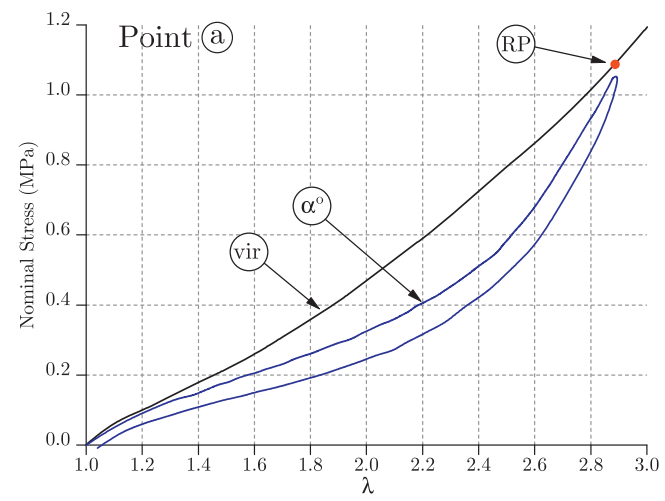

(a)

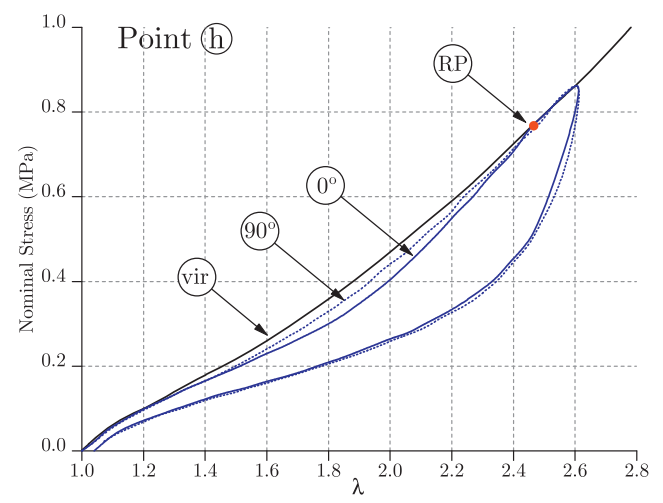

(c)

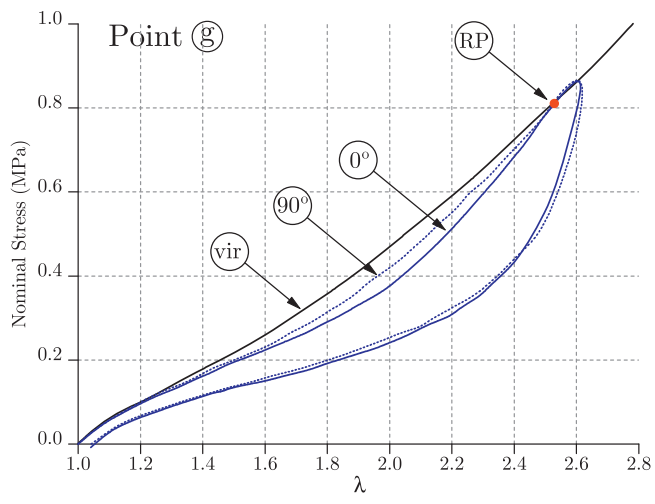

(b)

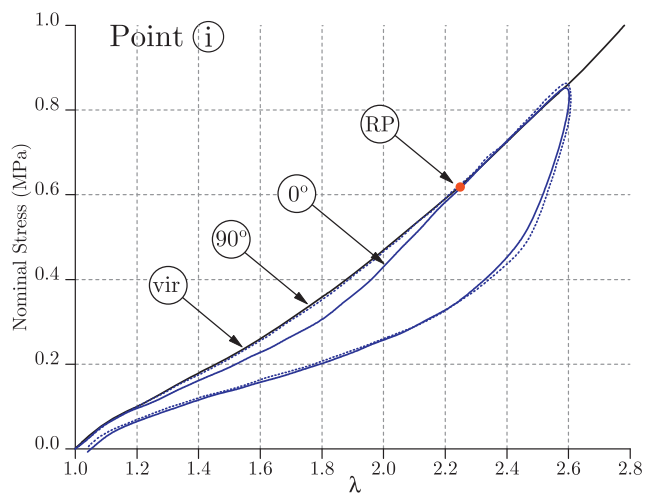

(d)

Fig. 6. Second uniaxial tensile load (and unload) curves of specimens $a, g, h$ and $i$ cut from the bulge plate at $0^{\circ}$ and $90^{\circ}$ with respect to the meridional direction. Note that, for point $a$, only one curve is presented since the all directions are equivalent.

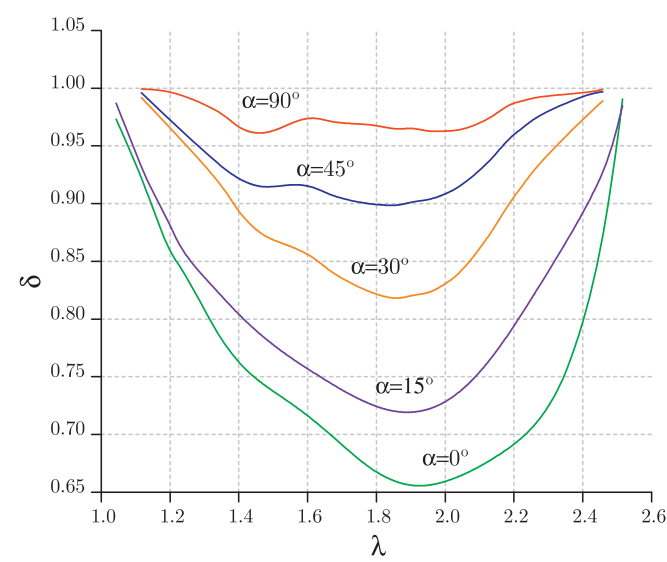

(a)

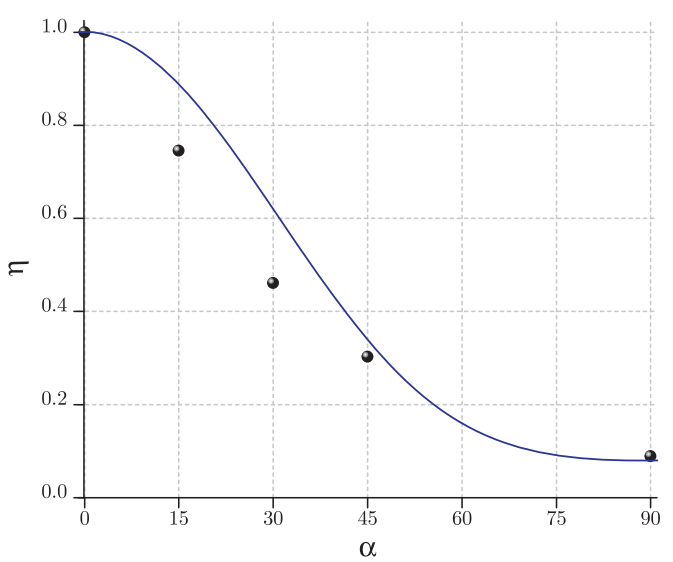

(b)

Fig. 7. (a) Stress ratio between the virgin uniaxial tensile load curve and the set of subsamples orientated at $0^{\circ}, 30^{\circ}, 45^{\circ}$ and $90^{\circ}$ (perpendicular) directions. (b) Ratio of energy loss according to the different loading angles.

reached tensile stretch $\left(\lambda_{\max }=2.50\right)$. The second load $\mathcal{W}_{2^{\text {nd }}}^{\left(0^{\circ}\right)}$ is used as reference since the same direction is used for the first and the second loading curves. Using a periodic approximation, the function $\eta$ in Eq. (6), is fitted by $\eta=a_{0}+a_{1} \cos (2 \alpha)+a_{2} \cos (4 \alpha)$

where $a_{0}=0.44, a_{1}=0.46$ and $a_{2}=0.10$. It appears that the energy loss ratio $(\eta)$ evolves from a maximum, when 


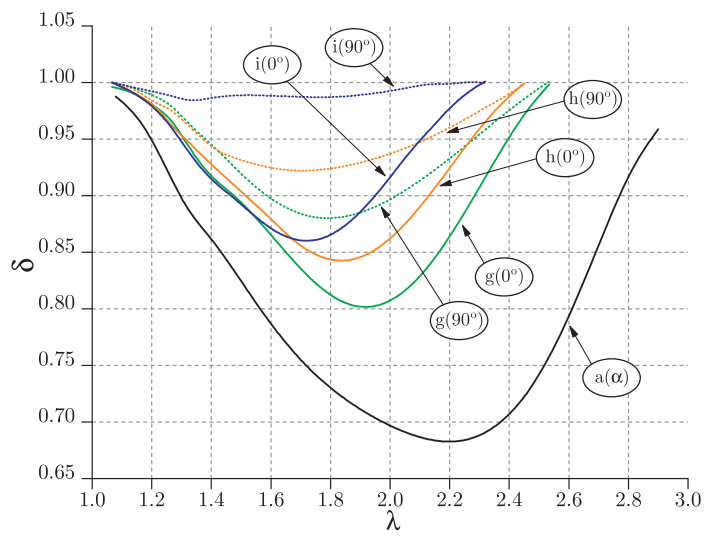

Fig. 8. Stress ratio between the virgin uniaxial tensile load curve (vir) and the second load for the uniaxial extension, performed on meridional $\left(0^{\circ}\right)$ and circumferential $\left(90^{\circ}\right)$ specimens, for points $a, g, h$, and $i$.

first and second tensile loadings are in the same direction to a minimum, close to zero, when the directions of first and second loadings are orthogonal.

\subsection{Anisotropy induced by biaxial tensile pre-stretch}

Concerning the biaxial tensile test preconditioning, Fig. 8 shows the uniaxial tensile test stress ratios of specimens cut in meridional $\left(0^{\circ}\right)$ and circumferential $\left(90^{\circ}\right)$ directions in the bulge specimen with respect to the uniaxial tensile test virgin curve (vir). Similiarly to Section 3.1, no proportionality between the first and second load curves was observed. Nevertheless, a main difference appears, compared with Fig. 7 . The curves at $90^{\circ}$ for points $g$ and $h$ present a considerable stress softening. This is due to the biaxial tensile pre-loading where the directional intensity of the stress softening depends on the biaxiality ratio $(\mu)$. This emphasizes that subsequent directional behavior is strongly influenced by how the maximal preloading is applied.

\subsection{Relevance of maximal deformation criterion for describing Mullins effect}

The choice of the Mullins effect evolution criterion is fundamental for the description of different loading paths. Considering the deformation of the material as a thermodynamic process, this criterion must describe the momentary maximum condition of the material deformation regardless of the path by which the system goes from one state to another. In other words, this variable defines how the stress softening evolves for an arbitrary path with respect to the maximum state achieved in the previous load path.

In the uniaxial tensile pre-stretch case, Fig. 1(b) showed that all second uniaxial tensile load curves come back on the first load curve at the same point $(R P)$, characterized by its tensile stretch, independently of the direction of the second load. For other pre-stretch load types than uniaxial tension, the maximum value of the major principal stretch is important to define the return point $(R P)$ but deformations in other directions cannot be neglected in stress softening accounting. Machado et al. (2010) showed that for an isotropic approach, the strain energy or the first invariant can be used to describe the evolution of stress softening. Fig. 5 which presents the second load curves after the biaxial tensile pre-stretch is replotted according to the first invariant in Fig. 9(a). In the same figure, the maximal values of $I_{1 \text { (max) }}$ reached during the biaxial preconditioning load from Fig. 4(a) have also been plotted. For each point $a$ to $j$, the values of the return point $\left(I_{1(R P)}\right)$, i.e., where the second load curves come back on the first uniaxial loading one (vir curve), are compared to the $I_{1 \text { (max) }}$. The results are presented in Fig. 9(b). The points do not fit well the first bisectrix. The bisectrix represents the one-to-one relationship between the first invariant for two different loading paths. It means that the first invariant cannot be a efficient criterion to describe the maximal deformation.

\subsection{Relevance of maximal strain energy criterion for describing Mullins effect}

The above results highlight that the maximal value of a thermodynamic variable reached in the loading history based on deformation measures are not sufficient as the amount of stress softening varies for a same maximal previous deformation. The fact that the material was loaded in the transverse direction in the biaxial pre-stretching, has a large influence on the stress softening, that means that a three dimensional criterion must be taken into account.

The Mullins effect can be quantified by the ratio between the strain energy during first loading $\left(\mathcal{W}_{0}\right)$ and the second loading $\left(\mathcal{W}_{2^{\text {nd }}}\right)$, defined as

$\psi=\frac{\mathcal{W}_{2^{n d}}}{\mathcal{W}_{0}}$

These strain energy densities are evaluated by integration of first and second load curves for both preconditioning methods. Note that, in the biaxial tension preconditioning, $\mathcal{W}_{0}$ is evaluated during the bulge test (for each point $a$ to $h$ ), whereas $\mathcal{W}_{2^{\text {nd }}}$ is evaluated from the second uniaxial tensile tests up to the return point limit. Hence, the ratio of strain energy is calculated and presented in Fig. 10. Furthermore, the cyclic tests results of uniaxial, planar and equibiaxial tensions, published in Machado et al. (2010), were extracted and superposed in the same plot composing an additional information about different loading paths. It clearly appears that all points obtained by different strain histories follow a tendency and they can be fitted by a master curve. A exponential decay approximation is proposed for the function $(\psi)$, and given by

$\psi=\psi_{o s}+\psi_{0} \exp \left(-c \mathcal{W}_{0}\right)$,

where $\psi_{o s}=0.29, \psi_{0}=0.63$, and $c=0.75 \mathrm{~m}^{3} / \mathrm{MJ}$. Note, in Fig. 10 that the energy ratio $\psi$ evolves from a maximum, where even minor deformation starts the softening process, to a minimum that cannot be reached before the rupture of the material. However, this master function does not make sense when the second load energy $\left(\mathcal{W}_{2^{n d}}\right)$ is evaluated in a direction of uniaxial tension different than 


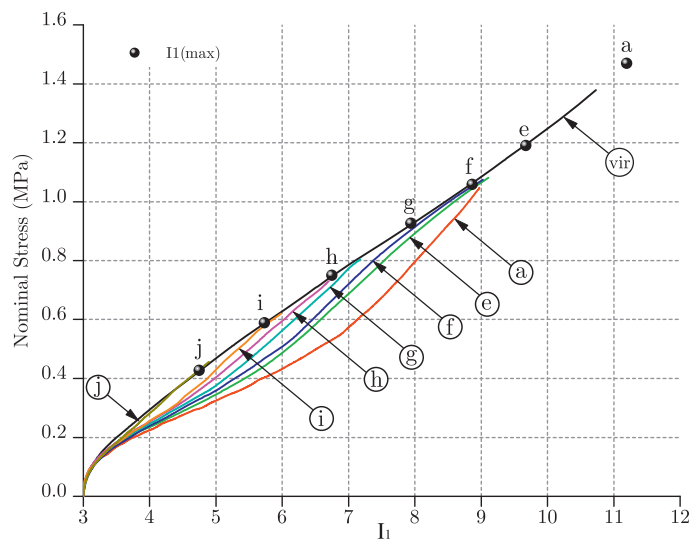

(a)

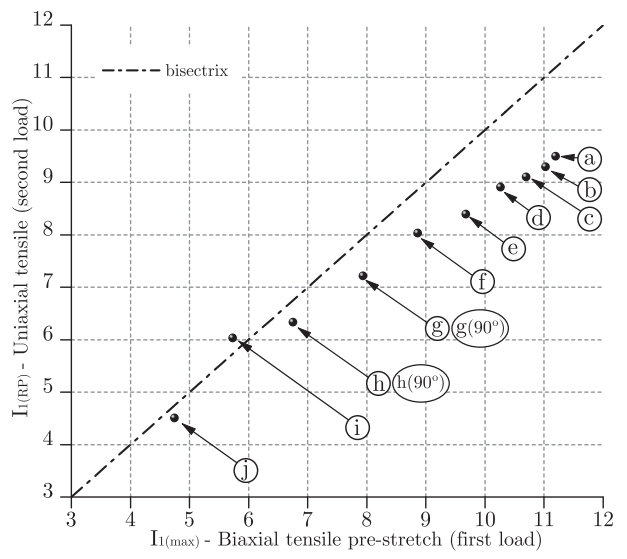

(b)

Fig. 9. Influence of the first biaxial tensile loading on uniaxial tensile test response. (a) Uniaxial tension performed at $0^{\circ}$ ) with respect to the meridional direction for points $\left(a, e, f, g, h, i\right.$, and $j$ - represented by solid lines) and the maximal first invariant $I_{1 \text { (max) }}$ (dots) reached during the biaxial first load (from Fig. 4(a)). (b) Relation between the maximal first invariant $I_{1(\max )}$ and the value of $I_{1(R P)}$ when the second load curve comes back on the reference first loading curve (vir-curve).

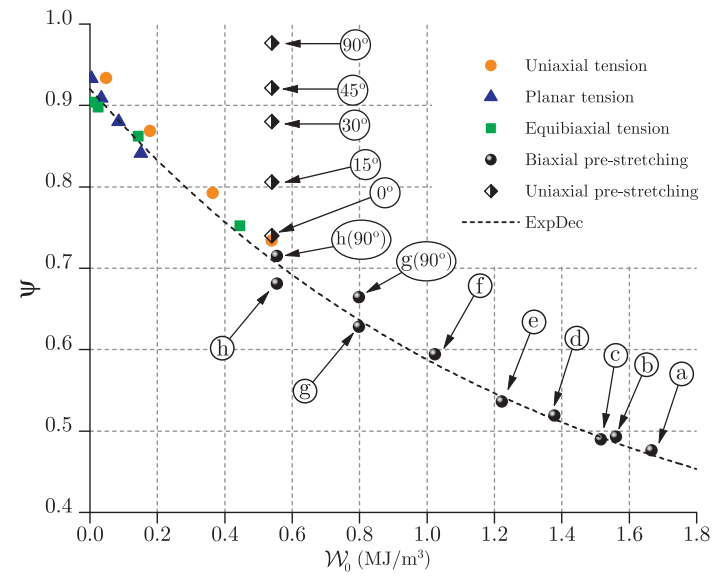

Fig. 10. Strain energy ratio $(\psi)$ between second loading (uniaxial tension) and different tensile first loadings. Points are fitted by a exponential decay function.

the pre-stretch principal direction of loading. The results from the uniaxial pre-stretching clearly show this noncompliance. In this manner, the strain energy must be investigated according to the strain principal directions of first and second loadings.

\subsection{Analysis of a decomposed strain energy}

Considering that the strain energy at first load in biaxial tension $\left(\mathcal{W}_{0}^{B}\right)$ can be splitted into two parts, then

$\mathcal{W}_{0}^{B}=\mathcal{W}_{0}^{(m)}+\mathcal{W}_{0}^{(c)}=\int P_{m} d \lambda_{m}+\int P_{c} d \lambda_{c}$,

where $\mathcal{W}_{0}^{(m)}$ and $\mathcal{W}_{0}^{(c)}$ are the strain energies at the first load for meridional and circumferential directions respectively. A schema is proposed in Fig. 11(a) to explain this splitting. Fig. 12(a) shows how these energy portions vary from the pole until the bulge border for the maximal pressure $(p=18 \mathrm{kPa})$. The meridional energy $\left(\mathcal{W}_{0}^{(m)}\right)$ decreases along the meridional direction but remains important, whereas the circumferential energy $\left(\mathcal{W}_{0}^{(c)}\right)$ tends to zero near the specimen border where there is no circumferential deformation.

With the knowledge of the above decomposition, the suitability of the parameter $\left(\mathcal{W}_{0}\right)$ to describe the stress softening can finally be evaluated. The idea is to compare if two different first loading paths can produce an energetically equivalent deformation state. The schema proposed in Fig. 11(b) for a generic position ( $a$ to $h$ ) in the bulge surface helps to explain this evaluation. First, the return points $(R P)$ of second uniaxial tensile loads on the uniaxial virgin tensile curve (vir) for each subsample $a$ to $h$ are identified. It is important to remind that, for a given position in the bulge surface, the return occurs at the same point $(R P)$ independently of the second tensile load direction as shown in Fig. 6. Then, an equivalent energy of a virgin material in uniaxial tension $\left(\mathcal{W}_{0}^{U}\right)$ is evaluated up to this same return point $R P$. Finally, this equivalent energy $\left(\mathcal{W}_{0}^{U}\right)$ is compared with the real energy $\left(\mathcal{W}_{0}^{B}\right)$ used to prestretch the membrane.

The strain energy $\mathcal{W}_{0}^{U}$ is plotted as function of $\mathcal{W}_{0}^{B}$ and the parts $\mathcal{W}_{0}^{(m)}$ and $\mathcal{W}_{0}^{(c)}$ in Fig. 12(b). The graph bisectrix represents the one-by-one relation between $\mathcal{W}_{0}^{U}$ and the other energies measures. It clearly appears that the total energy $\mathcal{W}_{0}^{B}$ values are not on the graph bisectrix. The fact that $\mathcal{W}_{0}^{B}$ is in the right side from the bisectrix means that a higher amount of energy would be necessary to produce the same state given by the $\mathcal{W}_{0}^{U}$ energy, i.e., defines the same $R P$ return on the virgin curve. In order words, there is a part of the total strain energy $\mathcal{W}_{0}^{B}$ that was not used in the stress softening process. Thus, the total strain energy cannot be the only key to describe the return on the virgin curve.

To measure the influence of loading directions, the same comparisons are realized but considering the decomposition proposed in Eq. (10). The Fig. 12(b) shows that the maximal principal direction strain energy, $\mathcal{W}_{0}^{(m)}$, fits better the bisectrix curve, while for the energy contri- 


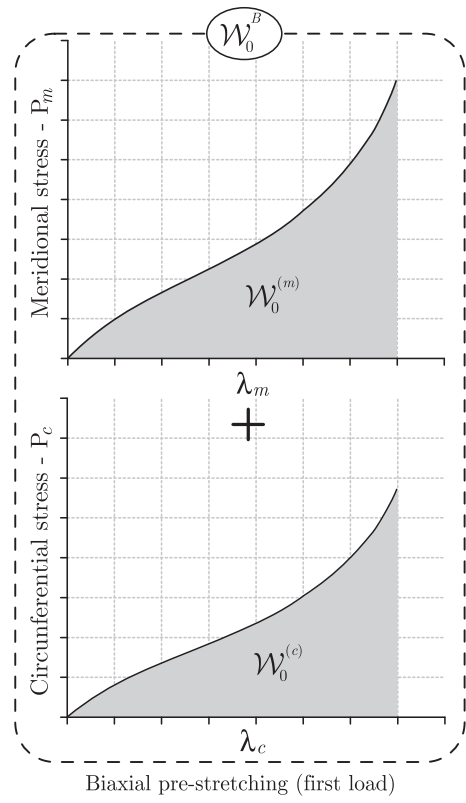

(a)

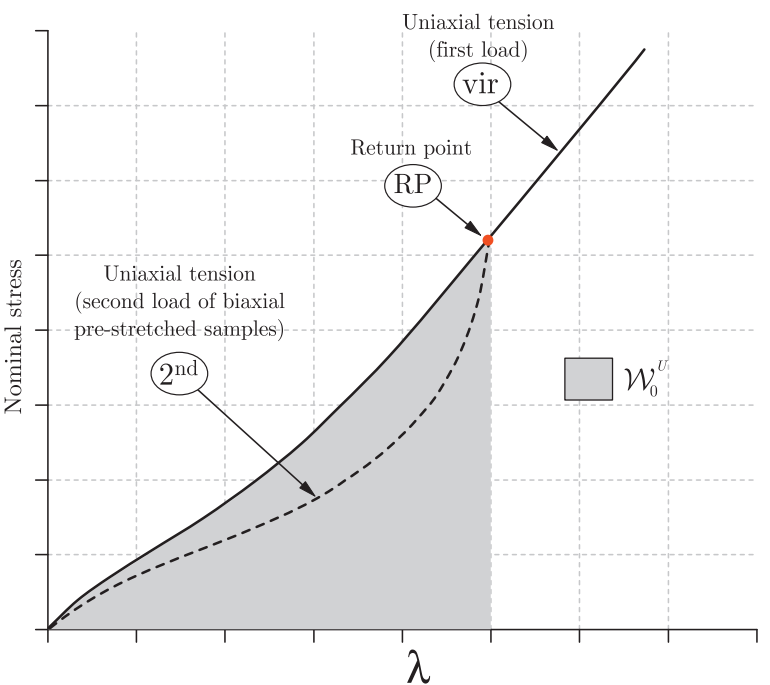

(b)

Fig. 11. First and second load strain energy schema. (a) The strain energy decomposition for the biaxial pre-stretching. (b) The equivalent energy ( $\left.\mathcal{W}_{0}^{U}\right)$ evaluated until the return point $(R P)$ of a given biaxial pre-stretched sample on the uniaxial first load reference curve (vir).

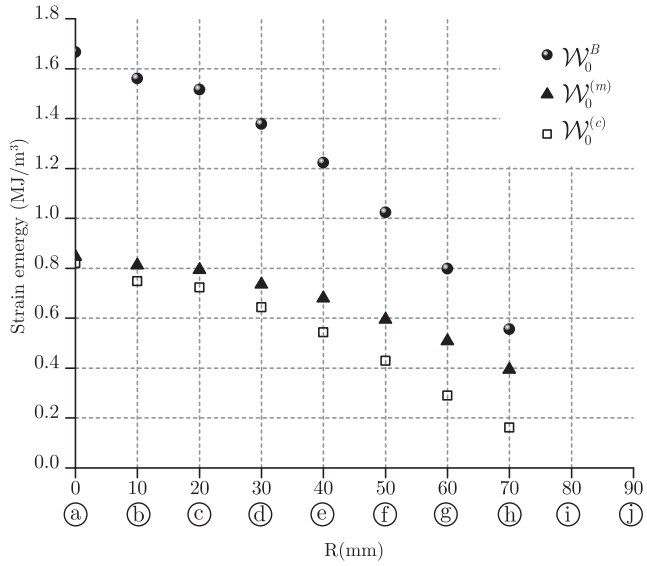

(a)

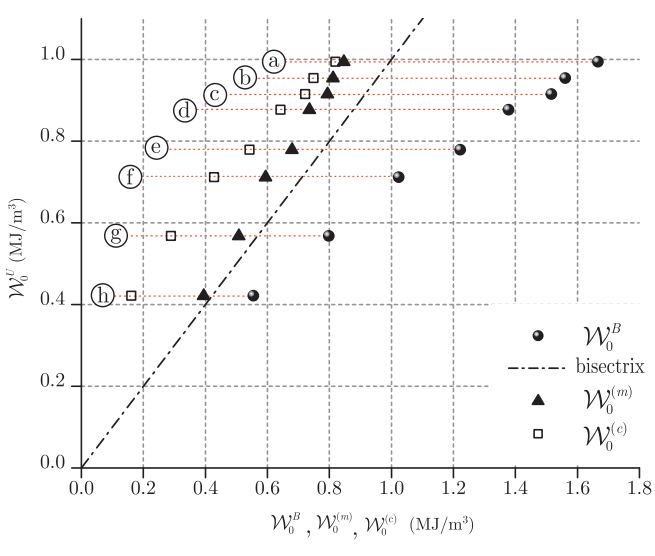

(b)

Fig. 12. Bulge test strain energy analysis.

bution in the other principal direction, $\mathcal{W}_{0}^{(c)}$, nothing can be concluded. This means that, if the maximal principal direction remains the same during the first and second load cycle, the amount of strain energy can be a measure to quantify the Mullins effect in this direction. In the other directions, a coupling effect exists between different directions and it influences the Mullins effect amount.

\section{Conclusion}

The motivating key for this work was the absence of a comprehensive experimental study to build evidence showing that the Mullins effect in filled silicone rubbers is a strongly anisotropic phenomenon. Using the 3D-DIC method as a non-contact full-field optical measurement, a large strain level was achieved and two methods were employed to record an initial deformation history. The results showed that uniaxial tensile preconditioning cycle in one direction hardly causes any softening in the perpendicular direction. Nevertheless, all second uniaxial tensile load curves come back on the first tensile load curve at the same point independently of the direction of the second load. The results with respect to the influence of Mullins effect in the intermediate directions permit to examine in detail the angular distribution of memory effects for testing and calibrating numerical models. 
In a original way, the bulge test was used as a biaxial tensile preconditioning test. The measurements made along the meridian of the inflated membrane provide unprecedented information about the history of principal stress and principal strain values without an explicit dependency of any constitutive relation. Results showed that the induced anisotropy is dependent on the prestretch biaxiality ratio. Again, the return on the first uniaxial tensile load reference curve of all second uniaxial tensile load curves occurs at the same point, independently of the second load direction. On one hand, the ratio of energy between first and second loading seems to follow a master behavior according to virgin strain energy. On the other hand, the come back on the virgin uniaxial curve can be controlled by the amount of energy, but only in the maximal principal direction whereas in the other directions, coupling phenomena exist between different directions.

Note also that the form and intensity of the anisotropy of the Mullins effect could depend on the elastomer type, the properties of filler particles, and their mass and volume proportion in the polymeric matrix.

\section{Acknowledgement}

We would like to thank the French ANR for supporting this work through the project RAAMO ("Robot Anguille Autonome pour Milieux Opaques").

\section{References}

Arruda, E.M., Boyce, M.C., 1993. A three dimensional constitutive mode for the large stretch behavior of rubber elastic materials. J. Mech Phys. Solids 41 (2), 389-412.

Besdo, D., Ihlemann, J., Kingston, J., Muhr, A. 2003. Modelling inelastic stress-strain phenomena and a scheme for efficient experimental characterization. In: Busfield, Muhr (Eds.), Constitutive models for Rubber III. Swets \& Zeitlinger, Lisse., pp. 309-317.

Chagnon, G., Verron, E., Gornet, L., Marckmann, G., Charrier, P., 2004. On the relevance of continuum damage mechanics as applied to the Mullins effect in elastomers. J. Mech. Phys. Solids 52, 1627-1650.

Diani, J., Brieu, M., Vacherand, J.M., Rezgui, A., 2004. Directional model isotropic and anisotropic hyperelastic rubber-like materials. Mech. Mater. 36, 313-321.

Diani, J., Brieu, M., Gilormini, P., 2006. Observation and modeling of the anisotropic visco-hyperelastic behavior of a rubberlike material. Int. J. Solids Struct. 43, 3044-3056.

Diani, J., Fayolle, B., Gilormini, P., 2009. A review on the Mullins effect. Eur. Polym. J. 45, 601-612.

Dorfmann, A., Ogden, R.W., 2004. A constitutive model for the Mullins effect with permanent set in particule-reinforced rubber. Int. J. Solids Struct. 41, 1855-1878.

Ehret, A.E., Itskov, M., 2009. Modeling of anisotropic softening phenomena: application to soft biological tissues. Int. J. Plast. 25 901-919.
Göktepe, S., Miehe, C., 2005. A micro-macro approach to rubber-like materials. Part III: The micro-sphere model of anisotropic Mullinstype damage. J. Mech. Phys. Solids 53, 2259-2283.

Govindjee, S., Simo, J.C., 1991. A micro-mechanically continuum damage model for carbon black filled rubbers in corporating Mullins's effect. J. Mech. Phys. Solids 39 (1), 87-112.

Hanson, D.E., Hawley, M., Houlton, R., Chitanvis, K., Rae, P., Orler, E.B. Wrobleski, D.A., 2005. Stress softening experiments in silica-filled polydimethylsiloxane provide insight into a mechanism for the Mullins effect. Polymer 46 (24), 10989-10995.

Horgan, C.O., Ogden, R.W., Saccomandi, G., 2004. A theory of stress softening of elastomers based on finite chain extensibility. Proc. $\mathrm{R}$. Soc. London A 460, 1737-1754.

Itskov, M., Haberstroh, E., Ehret, A.E., Vohringer, M.C., 2006. Experimental observation of the deformation induced anisotropy of the Mullins effect in rubber. KGK-Kautschuk Gummi Kunststoffe 59 (3), 93-96.

Itskov, M., Ehret, A., Kazakeviciute-Makovska, R., Weinhold, G., 2010. A thermodynamically consistent phenomenological model of the anisotropic Mullins effect. ZAMM - Journal of Applied Mathematics and Mechanics/ Zeitschrift für Angewandte Mathematik und Mechanik 90 (5), 370-386.

Johnson, M.A., Beatty, M.F., 1993. The Mullins effect in uniaxial extension and its influence on transverse vibration of rubber string. Continuum Mech. Thermodyn. 5, 83-115.

Laraba-Abbes, F., Ienny, P., Piques, R., 2003. A new tailor-made methodology for the mechanical behaviour analysis of rubber-like materials: II. Application to the hyperelastic behaviour characterization of a carbon-black filled natural rubber vulcanizate Polymer 44 (3), 821-840.

Machado, G., Chagnon, G., Favier, D., 2010. Analysis of the isotropic models of the Mullins effect based on filled silicone rubber experimental results. Mech. Mater. 42 (9), 841-851.

Machado, G., Favier, D., Chagnon, G. 2012. Membrane curvatures and stress-strain full fields of axisymmetric bulge tests from 3D-DIC measurements. Theory and validation on virtual and experimental results. Exp. Mech., http://dx.doi.org/10.1007/s11340-011-9571-3.

Marckmann, G., Verron, E., Gornet, L., Chagnon, G., Fort, P.C.P., 2002. A theory of network alteration for the Mullins effect. J. Mech. Phys. Solids. 50, 2011-2028

Meunier, L., Chagnon, G., Favier, D., Orgéas, L., Vacher, P., 2008. Mechanical experimental characterisation and numerical modelling of an unfilled silicone rubber. Polym. Test. 27, 765-777.

Muhr, A.H., Gough, J., Gregory, I.H. 1999. Experimental determination of model for liquid silicone rubber: Hyperelasticity and Mullins effect. In: Proceedings of the First European Conference on Constitutive Models forRubber 181-187. Dorfmann A. Muhr A.

Mullins, L., 1947. Effect of stretching on the properties of rubber. J. Rubber Res. 16, 275-289.

Ogden, R.W., Roxburgh, D.G., 1999. A pseudo-elastic model for the Mullins effect in filled rubber. Proc. R. Soc. Lond. A 455, 2861-2877.

Papkov, V.S., Godovskii, Y.K., Bulkin, A.F., Zhdanov, A.A., Slonimskii, G.L., Andrianov, K.A., 1975. Energy investigation of the softening of siloxane rubbers during deformation. Mech. Composite Mater. 11 (3), 329-333.

Park, B.H., Hamed, G.R., 2000. Anisotropy in gum and black filled sbr and nr vulcanizates due to large deformation. Korea Polymer J. 8, 268275.

Pawelski, H. 2001. Softening behaviour of elastomeric media after loading in changing directions. Constitutive models for rubber, Besdo, Schuster \& Ihleman (Eds.), pp. 27-34.

Rigbi, Z., 1980. Reinforcement of rubber by carbon black. Adv. Polym. Sci. 36, 21-68.

Shariff, M.H.B.M., 2006. An anisotropic model of the Mullins effect. J. Eng. Math. 56 (4), 415-435. 